\title{
Confinement Effects on an Electron Transfer Reaction in Nanoporous Carbon Electrodes
}

\author{
Zhujie $\operatorname{Li}^{1,2,3}$, Guillaume Jeanmairet ${ }^{2,3}$, Trinidad Méndez-Morales ${ }^{1,2,3}$, \\ Mario Burbano ${ }^{1,3}$, Matthieu Haefele ${ }^{1}$, Mathieu Salanne $e^{1,2,3}$ \\ ${ }^{1}$ Maison de la Simulation, CEA, CNRS, Univ. Paris-Sud, \\ UVSQ, Université Paris-Saclay, F-91191 Gif-sur-Yvette, France \\ ${ }^{2}$ Sorbonne Universités, UPMC Univ Paris 06, CNRS, \\ Laboratoire PHENIX, F-75005 Paris, France and \\ ${ }^{3}$ Réseau sur le Stockage Électrochimique de l'Énergie (RS2E), FR CNRS 3459, Franc£*
}

\begin{abstract}
Nanoconfinement generally leads to drastic effect on the physical and chemical properties of ionic liquids. Here we investigate how the electrochemical reactivity in such media may be impacted inside nanoporous carbon electrodes. To this end, we study a simple electron transfer reaction using molecular dynamics simulations. The electrodes are held at constant electric potential by allowing the atomic charges on the carbon atoms to fluctuate. We show that the $\mathrm{Fe}^{3+} / \mathrm{Fe}^{2+}$ couple dissolved in an ionic liquid exhibits a deviation with respect to Marcus theory. This behavior is rationalized by the stabilization of a solvation state of the $\mathrm{Fe}^{3+}$ cation in the disordered nanoporous electrode that is not observed in the bulk. The simulation results are fitted with a recently proposed two solvation state model, which allows us to estimate the effect of such a deviation on the kinetics of electron transfer inside nanoporous electrodes.
\end{abstract}

Nanoconfinement effects strongly impact on many liquid properties, such as transport, diffusion coefficients, phase transitions, and solvation structures ${ }^{1 / 4}$. They are particularly important for supercapacitors, which have emerged as a complimentary energy storage solution to batteries $^{5}$. This is due to the fact that supercapacitors display faster charging times, and consequently higher power deliveries than batteries, while attaining longer life cycles. However, the energy density of batteries is higher. In supercapacitors energy storage is realized through the adsorption of ions at the surface of two oppositely polarized electrodes, forming a so-called electrical double layer ${ }^{6}$. Nanoconfinement has been observed to have a significant influence on the performance of supercapacitors, as has been demonstrated by experiments78 and simulations ${ }^{9}$, 12 , where it was shown that the use of materials with sub nanometric pores as electrodes greatly increase the capacitance of these devices.

Within the realm of electrochemical applications, room temperature ionic liquids (RTILs) have attracted a considerable attention ${ }^{13114}$. In principle, the properties of RTILs are tunable due to the wide variety of cations and anions from which they can be prepared. Because RTILs are solely made of charged species, it would have been expected that using them to replace standard electrolytes would increase the performance of supercapacitors. Yet, the interfacial capacitance of RTILs and acetonitrilebased electrolyte supercapacitors remain somewhat similar. This rather disappointing observation has been attributed to the more important correlations between ions in RTILs, which is due to the absence of electrostatic screening by the solvent ${ }^{15}$.

An alternative could be to take advantage of the tunability of RTILs to develop new storage concepts. Recently, Mourad et al. reported a large enhancement of the energy stored due to simultaneous capacitive and Faradic processes when biredox RTILs are used as electrolytes in a supercapacitor $\frac{16}{16}$. Among the various questions raised by this study, the most important ones are: How is the electron transfer rate affected by confinement? Does the charging of such a supercapacitor remain dominated by the ionic diffusion?

From the theoretical point of view, electron transfer reactions in solution are usually studied in the framework of Marcus theory, which aims at accounting for the influence of solvent fluctuations on the rate of electron transfer 17 . Marcus theory has been widely used to interpret experiments and simulations with an undeniable success. However, some systems exhibiting a deviation from Marcus linear behavior have been reported 18119 . Several extensions to the theory were proposed to account for them20121. A reason why Marcus theory might fail arises from the fact that one of its key assumptions is that the fluctuations of the solvent around the reactant and the product are similar. Yet, if the solvation states of the two species are structurally different, this hypothesis can be wrong as evidenced by the use of density functional theory-based Molecular Dynamics simulations of aqueous copper and silver ions 1921 . In experiments as well, introducing asymmetry in Marcus theory ${ }^{\sqrt{22}}$ was necessary to fit the voltammetric data of various RTILs 23 . Finally, in the case of interfacial systems several studies have underlined the importance of field penetration into the metal and of solvent spatial correlations. ${ }^{24 / 25}$

Confinement might have a drastic influence on the solvation shell of the adsorbed species ${ }^{26 \mid 27}$. Thus, it is worth investigating this effect on the electron transfer rate 28 . We report here a molecular dynamics study of the $\mathrm{Fe}^{3+} / \mathrm{Fe}^{2+}$ electron transfer reaction which has already been proposed in previous redox supercapacitor concepts 29130 . The experimental studies involve complex species, so that we focus on a simplified system in order to gain a first insight on electron transfer reactions in carbon nanopores. In particular, our study corresponds 
to infinite dilution since it is known that inorganic salts have low solubilities in imidazolium-based RTILs 31 . The studied ionic liquid is the 1-ethyl-3-methylimidazolium tetrafluoroborate $\left(\mathrm{EMIM}-\mathrm{BF}_{4}\right)$, which is put in contact with model carbide-derived carbon (CDC) nanoporous electrodes. We show that the free energy profiles for the electron transfer reaction strongly deviate from Marcus theory due to the presence of two solvation states for the $\mathrm{Fe}^{3+}$ species.

We build on previous work by using a simulation cell similar to the one we used to investigate the origin of increased capacitance in nanoporous carbon-based supercapacitors 10 . We employ a coarse-grained model of the EMIM-BF ${ }_{4}$ with, respectively, 3 and 1 interaction sites for the cation and the anion $\frac{33}{3}$, to which we add one iron ion and the appropriate number of chloride counter ions. We keep the electric potential inside the electrodes constant and equal to $0 \mathrm{~V}$ by using the procedure developed by Sprik et al. ${ }^{34}$, which allows the charge on the electrode to adjust in response to the local electric potential due to the electrolyte ions. Each electrode is represented by a model of CDC containing 3821 carbon atoms 35 . To address the effect of the local environment experienced by the redox species we ran several simulations in which the initial position of the iron ion is set in different pores of the disordered carbon material. In order to study the following redox half-reaction:

$$
\mathrm{Fe}^{2+} \rightarrow \mathrm{Fe}^{3+}+\mathrm{e}^{-}
$$

we performed simulations of the reduced species $\mathrm{Fe}^{2+}$ and of the oxidized one $\mathrm{Fe}^{3+}$. It has been shown by Warshe ${ }^{36 \mid 37}$ that a relevant reaction coordinate to study electron transfer reaction is the vertical energy gap $\Delta E$, which is defined as

$$
\Delta E\left(\left\{\mathbf{R}^{N}\right\}\right)=E_{1}\left(\left\{\mathbf{R}^{N}\right\}\right)-E_{0}\left(\left\{\mathbf{R}^{N}\right\}\right)
$$

where $E_{1}$ and $E_{0}$ are the instantaneous potential energies of a system with either the reduced or the oxidized state of the redox-active species for a given microscopic configuration $\left\{\mathbf{R}^{N}\right\}$. We recall here that in the linear response assumption in Marcus theory ${ }^{17 / 38}$, the distribution of the order parameter is Gaussian $\frac{39}{39}$ and, in particular, the shape of the distribution of $\Delta E$ obtained by performing simulations with the Hamiltonian of the reactant or the product should be identical. As shown in 2 a, this is clearly not the case, the distribution for the $\mathrm{Fe}^{2+}$ being almost Gaussian, while the one for the $\mathrm{Fe}^{3+}$ is not. This is a first indicator that this system does not follow Marcus theory picture.

In order to increase the statistical accuracy, we use the free-energy perturbation method. This variant of umbrella sampling introduces a coupling parameter $(\eta)$ between the reduced and oxidized states ${ }^{40}$. The simulations are then performed with an intermediate Hamiltonian associated to the potential energy surface $E_{\eta}$ defined as the linear superposition:

$$
E_{\eta}=(1-\eta) E_{0}+\eta E_{1}
$$

A direct consequence of the linear assumption of Marcus theory is that $E_{\eta}$ should vary linearly with $r_{i}^{40}$. To test this, we carried out simulations with different values of the coupling parameter $\eta=0,0.25,0.5,0.75,0.8,0.9$ and 1.0. It is clear for $2 \mathrm{~b}$ that the Marcus picture is violated, since the corresponding vertical energy gaps do not follow the expected linear variation.

As pointed out in the introduction, discrepancy with Marcus theory often originates from strong changes in the solvation shell between the reduced and oxidized states. To investigate if that is indeed the case here, we examine the coordination number of the iron cation along the simulations. The radius of the first solvation shell is taken equal to the first minimum of the $\mathrm{Fe}_{-} \mathrm{BF}_{4}^{-}$radial distribution function in the absence of electrodes $(3.8 \AA)$. We observe that the coordination number $(\mathrm{CN})$ of the $\mathrm{Fe}^{3+}$ cation fluctuates between values of 4 and 6 during the simulation. This is clearly an effect of the confinement since the cation remains close to the carbon electrode, and the corresponding $\mathrm{CN}$ is 4 in the pure RTIL. In contrast, the $\mathrm{Fe}^{2+}$ cation has a $\mathrm{CN}$ that remains equal to 4 during the whole simulations, as shown in Supplementary Figure S1.

The distances between $\mathrm{Fe}^{3+}$ and the six nearest anions along a representative trajectory are displayed in $3 \mathrm{a}$; the other simulations display similar variations. The alternation between two coordination environments is confirmed by the fact that during some parts of the simulations we detected that 4 anions are closely coordinated to the $\mathrm{Fe}^{3+}$ with a $\mathrm{Fe}-\mathrm{BF}_{4}$ distance of $2.8 \AA$, the two remaining anions being located further away from the iron with a distance greater than $4 \AA$, while in other parts the 6 anions are coordinated with $\mathrm{Fe}^{3+}$ with metal-ligand distances fluctuating between 2.6 and $3.8 \AA$. 3 . and $3 \mathrm{~d}$ show representative snapshots of the two coordination states which appear to be tetrahedral and octahedral, respectively. The corresponding fluctuations of the vertical energy gap are shown on $3 \mathrm{p}$. Sudden changes occur concommitently with the jumps in $\mathrm{CN}$ in 3 a, which shows that the confinement effect on the solvation of $\mathrm{Fe}^{3+}$ is at the origin of the departure from Marcus theory discussed above.

Among all models available in the literature to extend Marcus theory to non-linear behavior ${ }^{41}$ we use the one proposed by Vuilleumier et al! 21 . In particular, we employed their two-Gaussian solvation (TGS) state model, which allows the reactant and product to experience two different solvation states $\left(S_{0}\right.$ and $\left.S_{1}\right)$. This leads to four (two pairs) diabatic free energy parabolas instead of two in the case of Marcus theory. For each of the states $\mathrm{S}_{0}$ or $\mathrm{S}_{1}$, the Landau free energy is assumed to be quadratic, which corresponds to a Gaussian probability distribution $P_{\eta}$. The authors also derived the necessary equations to compute the average energy gap $\langle\Delta E\rangle_{\eta}$ and the Landau free energies. 


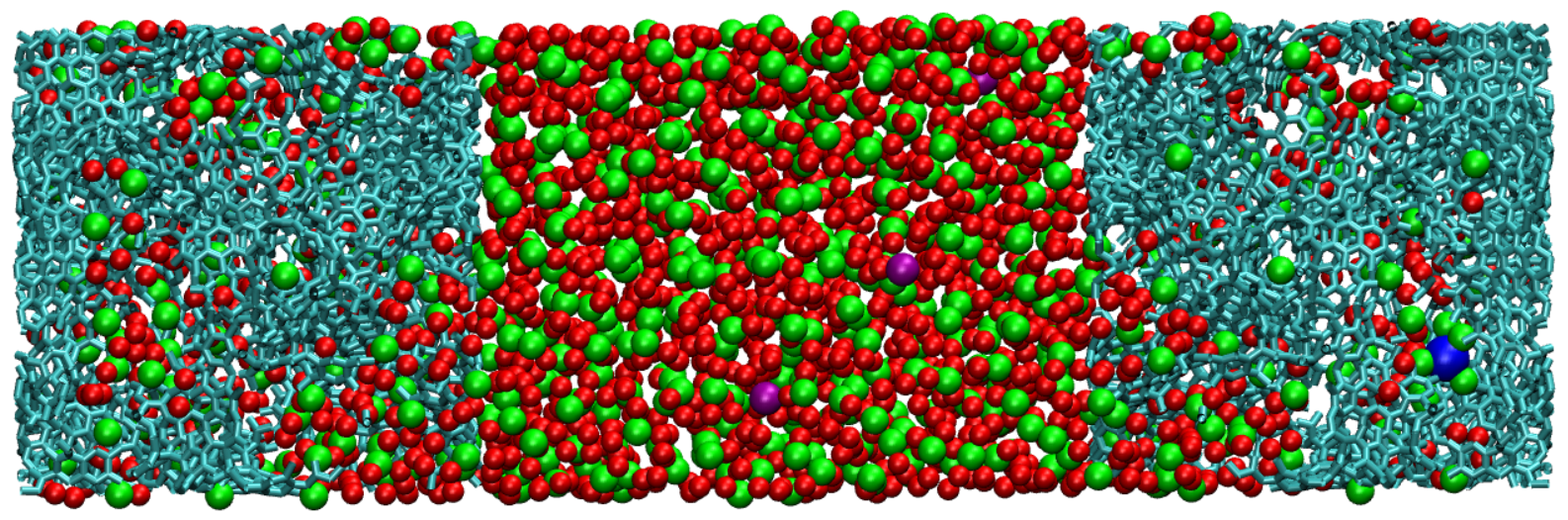

FIG. 1: The simulation cell is constituted of two porous electrodes held at constant potential (cyan C atoms). The electrolyte is composed of a $\mathrm{FeCl}_{3}$ or $\mathrm{FeCl}_{2}$ unit dissolved in EMIM-BF 4 , modeled using a coarse-grained force-field ${ }^{32}$ (red: the three interaction sites of $\mathrm{EMIM}^{+}$, green: the single site of $\mathrm{BF}_{4}^{-}$, blue: Fe (III) cation and violet: $\mathrm{Cl}^{-}$anions).
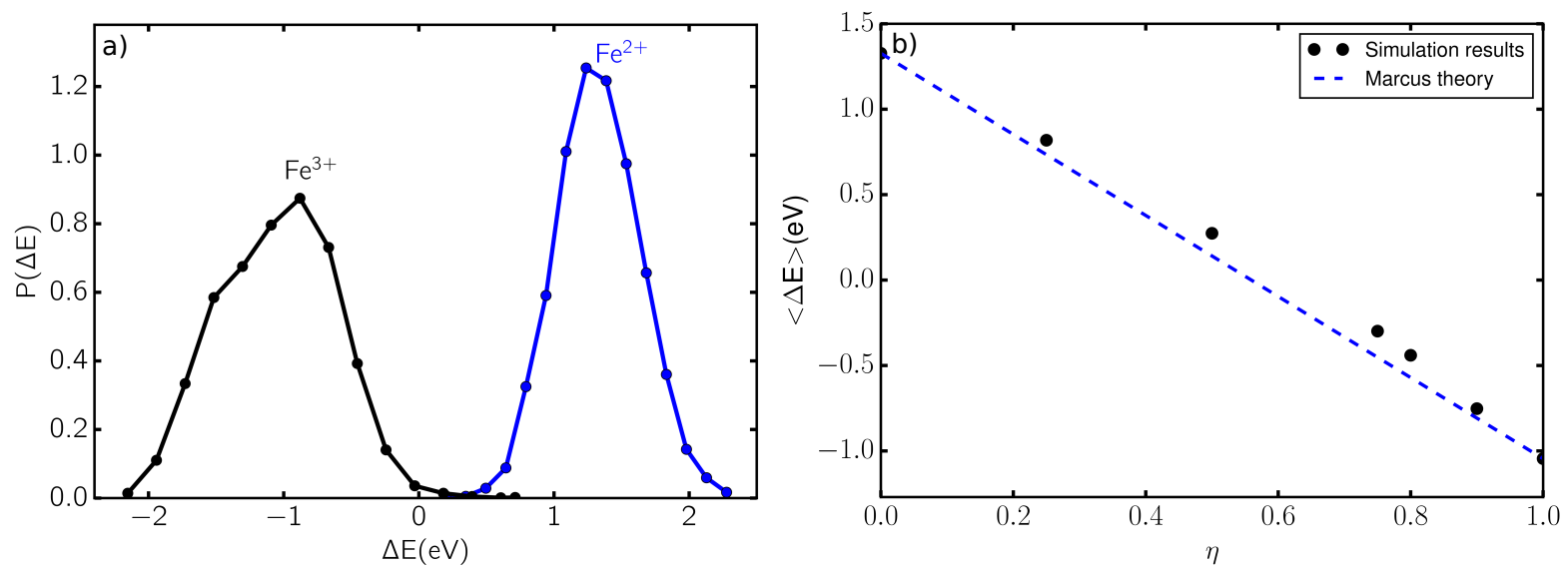

FIG. 2: a) Probability distribution of the vertical energy gap in reduced and oxidized states, b) Average vertical energy gap for $\mathrm{Fe}^{3+} / \mathrm{Fe}^{2+}$ in RTILs with various coupling parameters $\eta$.

Marcus theory involves only 2 parameters: $\Delta A$ measures the relative position of the two parabolas while $\lambda$ is the reorganization energy of the solvent, which fixes the curvature of the parabola and also the width of the Gaussian distribution of the reaction coordinate. In the TGS model, 4 parameters are required, one reorganization energy for each state $\lambda_{S_{0}}$ and $\lambda_{S_{1}}$ and the relative positions of the two parabola for each solvation state $\Delta A_{S_{0}}$ and $\Delta A_{S_{1}}$. Moreover, a fifth parameter is necessary to set the relative position of the two pairs of parabolas, for instance the difference in free energy between the two solvation states of the reduced species $\Delta_{S} A_{0}=A_{S_{1}}^{\mathrm{Fe}^{2+}}-A_{S_{0}}^{\mathrm{Fe}^{2+}}$.

The effect of the confinement was not investigated by the authors of the TGS model, since they only carried out simulations in bulk. However, it has been shown from simulation studies of the $\mathrm{Eu}^{3+} / \mathrm{Eu}^{2+}$ redox couple in potassium chloride molten salt at an fcc metallic electrode that the reorganization energy strongly depends on the distance between the ion and the electrode ${ }^{42}$. This reorganization energy is expected to decrease when the redox species get closer to the electrode. We mention that this behavior had previously been predicted theoretically by Marcus for perfect conductors, $\stackrel{38 \mid 43}{ }$ but this effect should decrease if the role of field penetration into the metal electrode was accounted.2425]

To quantify this effect in our system, we performed additional simulations with a planar graphite electrodes. They confirm the dependency on the distance to the electrode of the reorganization energy, as shown in Supplementary Figures S2 to S4. In nanoporous electrodes it is not possible to define a simple distance to the electrode, the fit of the TGS model should therefore yield an averaged contribution of all the possible distances. However we observed that the iron does not go into contact with the carbon during the whole simulation data, regardless of the iron solvation state, the nature of the pore in which it is solvated or its coordination number. For this reason and for the sake of simplicity we made the additional assumption that the reorganization energies should be equal for both states, turning the original 5-parameter model TGS into a 4 parameter-one. The different parame- 

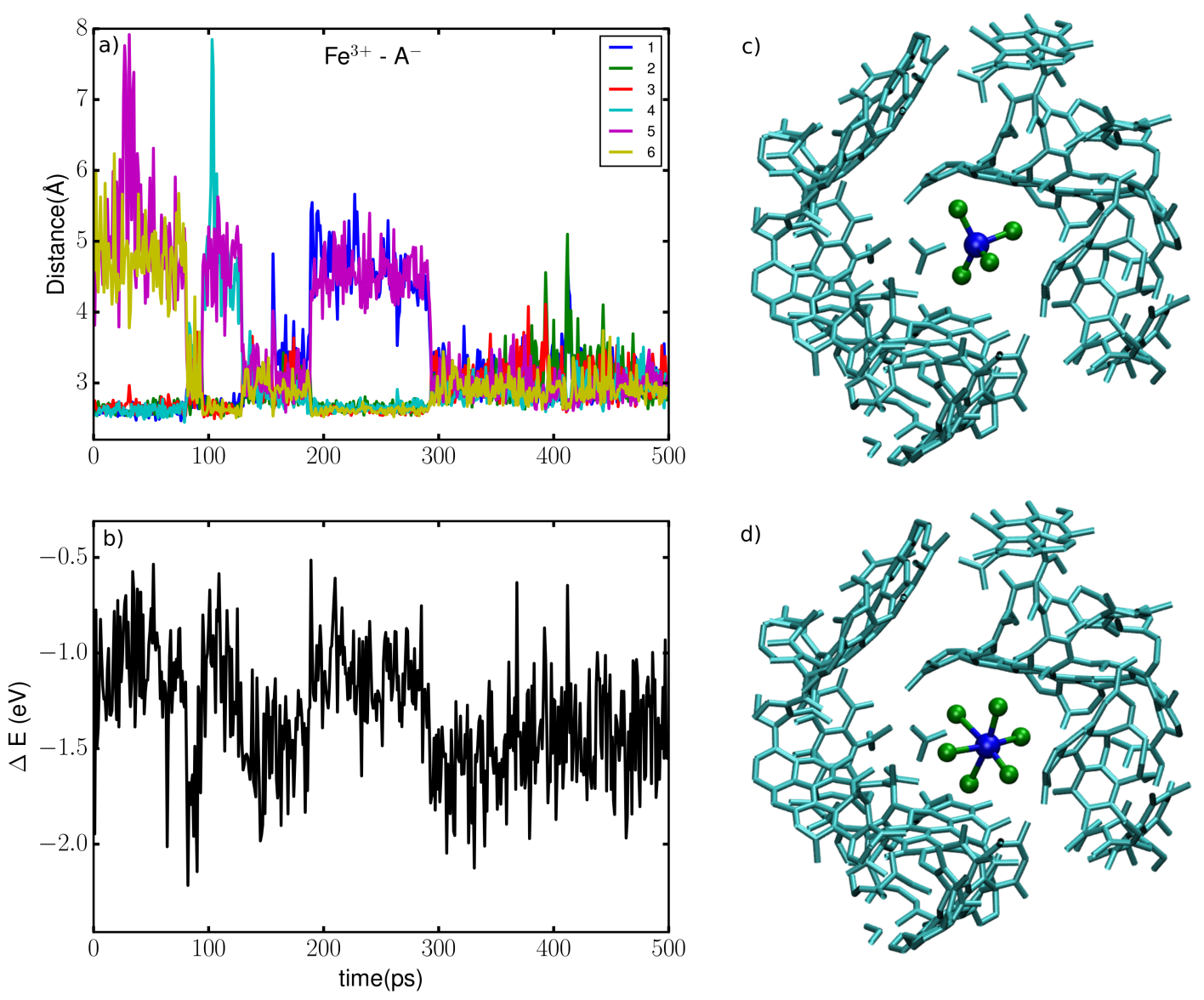

FIG. 3: a) Distance between $\mathrm{Fe}^{3+}$ and the six closest anions, b) Vertical energy gaps of the oxidized state Fe ${ }^{3+}$, c) and d) Snapshots of the two different solvation structures.

TABLE I: TGS model parameters for $\mathrm{Fe}^{3+} / \mathrm{Fe}^{2+}$ obtained by fitting simultaneously on $P_{\eta}(\Delta E)$ and $\langle\Delta E\rangle_{\eta}$.

\begin{tabular}{llllll}
\hline TGS parameters & $\lambda_{S_{0}}$ & $\Delta A_{S_{0}}$ & $\lambda_{S_{1}}$ & $\Delta A_{S_{1}}$ & $\Delta_{S} A_{0}$ \\
\hline$(\mathrm{eV})$ & 1.11 & 0.24 & 1.11 & -0.27 & 0.52 \\
\hline
\end{tabular}

ters obtained by fitting the simulation data are given in [

4 compares the results obtained by simulations to the TGS model with the parameters given in The left hand panel shows the probability distribution of the vertical energy gap, for the simulations realized with different values of the $\eta$ parameter. The fitted TGS model agrees well with the whole set of distribution. The right hand panel reproduces the data of 2 i.e. the vertical energy gap as a function of $\eta$. While those data could not be well reproduced by assuming linear response, the use of the TGS model allows an almost perfect fit to the data.

The diabatic free energy curves for the reduced and oxidized species in $\mathrm{Fe}^{3+} / \mathrm{Fe}^{2+}$ predicted by the TGS model with the parameters of $\mathrm{I}$ are plotted in 5 . The two parabolas for the reduced or oxidized state $(\eta=0$ or 1) correspond to a given solvation state. The free en- ergy difference $\left(\Delta_{S} A_{0}\right)$ between the hexacoordinated and the tetracoordinated forms of $\mathrm{Fe}^{2+}$ is large $(0.52 \mathrm{eV})$ with respect to the thermal energy $k_{B} T(34.5 \mathrm{meV}$ at $T=400 \mathrm{~K})$. The hexacoordinated state is therefore very unlikely, which explains why it is never observed in our equilibrium simulations. On the other hand, the free energies of the two coordination states are very close from each other for $\mathrm{Fe}^{3+}$.

The thermodynamic and kinetic informations extracted from 5 are summarized in a mechanistic diagram in 6. We can see that the electron transfer from tetracoordinated $\mathrm{Fe}^{2+}$ to hexacoordinated $\mathrm{Fe}^{3+}$ is as likely as the transfer to the tetracoordinated $\mathrm{Fe}^{3+}$ from a thermodynamic point of view, since the corresponding free energy differences are very similar, 0.24 and $0.25 \mathrm{eV}$ respectively. From a kinetic point of view the picture is completely different since the corresponding activation energy, taken at the intersection between the two parabolas, is much higher $(0.55 \mathrm{eV}$ vs. $0.39 \mathrm{eV})$. As a consequence, the effect of the confinement is likely to have a negative impact on the kinetic rate of the electron transfer reaction. Indeed, it allows for the existence of hexacoordinated $\mathrm{Fe}^{3+}$, which is not stable in the bulk liquid in our simulations. The electron transfer from $\mathrm{Fe}^{2+}$ to this chemical species has a 

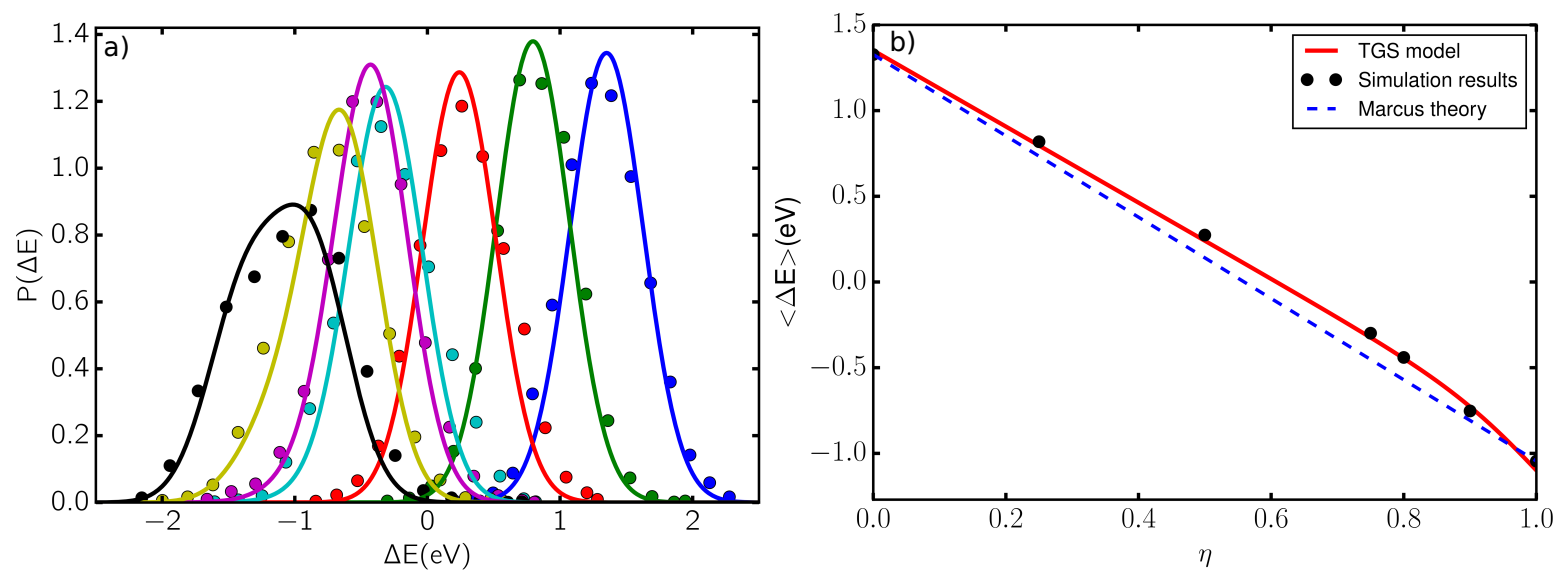

FIG. 4: a) Probability distribution of the vertical energy gap, as a function of the coupling parameter $\eta$. Points are the simulation data, and lines are the TGS model with the parameters from I b) Comparison between the simulated average vertical energy gap for $\mathrm{Fe}^{3+} / \mathrm{Fe}^{2+}$ in RTILs with various coupling parameters $\eta$ (points) and the TGS model (red line).

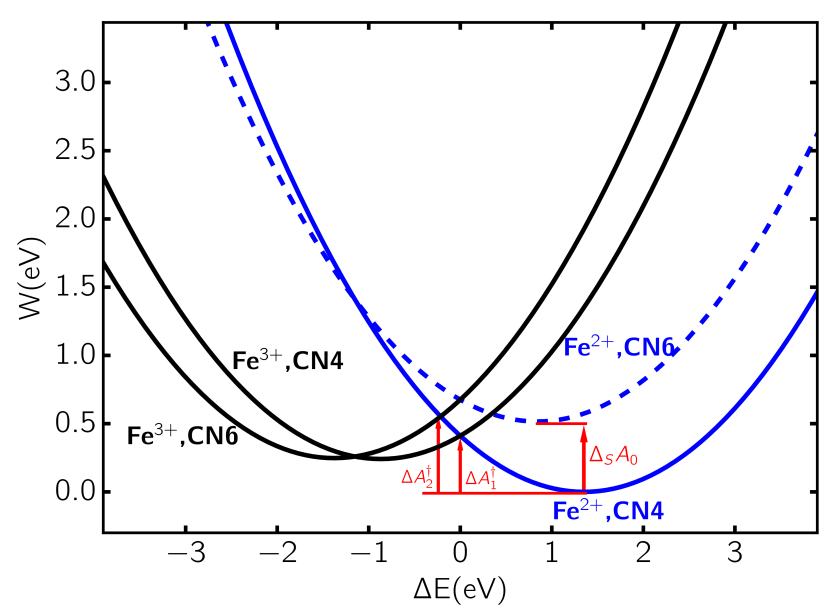

FIG. 5: Diabatic free energy curves of the reduced and oxidized species obtained by TGS model with the parameters of [. "CN4" and "CN6" labels refer to the tetra and hexacoordinated solvation states and correspond to $\mathrm{S}_{0}$ and $\mathrm{S}_{1}$ respectively.

very large activation energy barrier, making such events unlikely to occur. This result contrasts with the observation of Remsing et al. who observed an enhancement of the electron transfer due to the confinement $\frac{44}{4 n}$ a very different system. This is easily explained because in their work both the reduced and oxidized species where desolvated with respect to the bulk, while we observe the opposite behavior.

In conclusion, the aim of this work was to investigate the free energy properties of an electron transfer reaction in a nanoporous carbon electrode. We performed MD simulations of the $\mathrm{Fe}^{3+} / \mathrm{Fe}^{2+}$ redox couple dissolved in EMIM-BF 4 RTIL in contact with CDC electrodes. The latter were maintained at a constant potential of $0 \mathrm{~V}$ during the simulations. The vertical energy gap

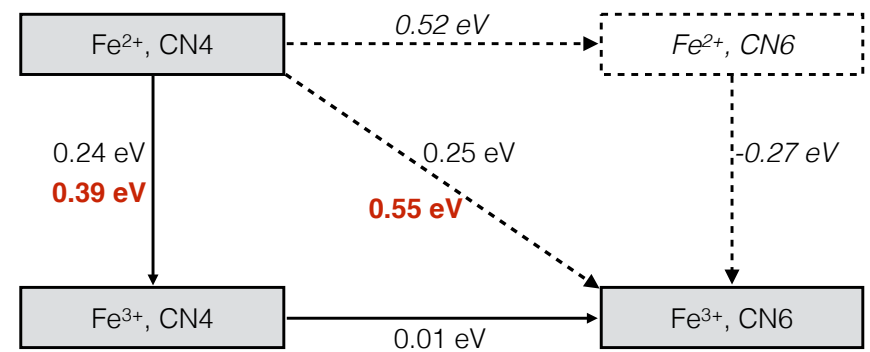

FIG. 6: Mechanistic diagram of the electron transfer reaction under confinement. Black numbers: free energy differences; red numbers: activation energies (labelled $\Delta A_{1}^{\dagger}$ and $\Delta A_{2}^{\dagger}$ on 5 .

between the redox species was used as the reaction coordinate, which enabled us to interpret the microscopic solvent fluctuation properties. The computed probability distribution of the vertical energy gaps and the equilibrium value of energy gaps do not follow the linear response approximation of standard Marcus theory. We demonstrated the strong influence of the nanoconfinement effect on the solvation shell of the iron cations, which is the main reason for this departure from Marcus theory. In particular, the $\mathrm{Fe}^{3+}$ cation, which is tetracoordinated in the bulk, admits two stable solvation states in the nanoporous material: tetracoordinated and hexacoordinated. To account for this deviation we used the two-Gaussian solvation model 21, from which the free energy curves for all the redox species in their various solvation states were extracted. This allowed us to qualitatively analyse the effect of the confinement on the electron transfer reaction. The fluctuations in the structure of the solvation shell of $\mathrm{Fe}^{3+}$ were shown to have a negligible effect from a thermodynamic point of view. Rather, it was shown that the activation energy of the associated electron transfer process is much higher for the hexacoordinated form. It is therefore 
likely that the stabilization of this solvation state will result in a slow down of the electron transfer reaction kinetic. This work is a first step towards a deeper understanding of the influence of the confinement on the electron transfer in redox supercapacitor devices ${ }^{29}$, which will be extended in future years to promising systems such as biredox RTILs 16 . To this end, it will be necessary to simulate more complex redox probes and to account systematically for the various complexation states. Another important aspect will be to decouple the contribution of the electron transfer event from the work-term that controls the approach of the ion to the interface in nanoconfinement ${ }^{28}$. The techniques develop in this work could also provide useful information for the development of ionic liquids-based thermo-electrochemical cells $\frac{45}{\text {. }}$.

\section{Methods}

We performed molecular dynamics (MD) simulations of the present systems using coarse-grained force fields. The non-bonded interactions are represented using Lennard-Jones and Coulomb potentials, and the corresponding parameters for carbon atoms and $\mathrm{EMIM}-\mathrm{BF}_{4}$ interaction sites we used herein are obtained from our previous work ${ }^{32 \mid 46]}$. The parameters for the chloride and the iron ions are respectively taken from references 47 and ${ }^{48}$ (the chloride anions were added in order to maintain the electroneutrality of the simulation cell, however their effect on the vertical energy gap was not studied since we focus on infinite dilution conditions). The simulations were conducted in the NVT ensemble, with the temperature set at $400 \mathrm{~K}$ using a Nosé-Hoover thermostat (relaxation time: $10 \mathrm{ps}$ ). The simulation cell is orthorhombic, with $x=y=4.37 \mathrm{~nm}, z=14.86 \mathrm{~nm}$, which reproduces the density of the RTILs compared with experimental results. Periodic boundary conditions are employed along $x$ and $y$ directions only through the use of a 2D-Ewald summation 49 . For all the simulations, the time step is $2 \mathrm{fs}$ and the time scales of productions are over 500 ps after equilibration. The electrodes are maintained at a constant potential ( $0 \mathrm{~V}$ in our present study) during the production runs. The vertical energy gap is sampled every 1 ps during the simulation. Following our previous work, a constant has been added to the energies calculated in the simulation to represent the (gas-phase) ionisation potential of $\mathrm{Fe}^{2+}$ and the work function of the metal 2 ; in practice, the value $(15.78 \mathrm{eV})$ was chosen to bring the calculated redox potential close to the typical experimental values for the $\mathrm{Fe}^{3+} / \mathrm{Fe}^{2+}$ couple in RTILs 5 .

\section{Supplementary figures}

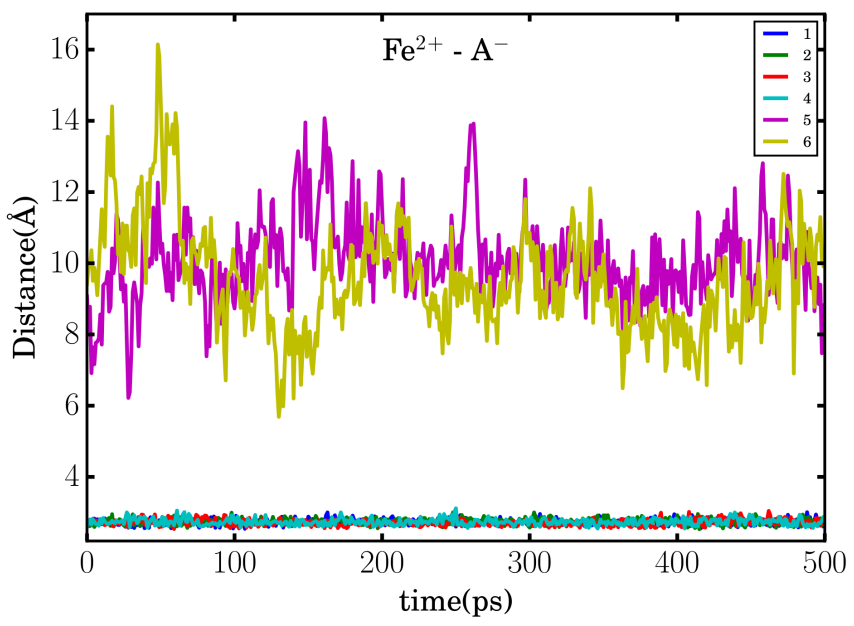

FIG. 7: The distance between selected anions $\left(\mathrm{A}^{-}\right)$and $\mathrm{Fe}^{2+}$ with respect to time, in $\mathrm{CDC}$ cell.

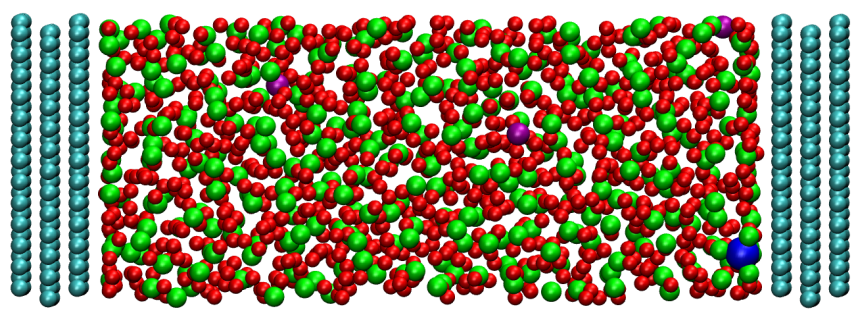

FIG. 8: The structure of simulation cell comprising EMIM$\mathrm{BF}_{4}$ ionic liquids and graphite planar electrodes.

\section{acknowledgement}

This work was supported by the French National Research Agency (Labex STORE-EX, Grant No. ANR10-LABX-0076). We acknowledge support from EoCoE, a project funded by the European Union Contract No. H2020-EINFRA-2015-1-676629, from the DSM-énergie programme of CEA and from the Eurotalent programme. We are grateful for the computing resources on OCCIGEN (CINES, French National HPC) and CURIE (TGCC, French National HPC) obtained through the project x2016096728.
* Electronic address: mathieu.salanne@upmc.fr

1 C Alba-Simionesco, B Coasne, G Dosseh, G Dudziak, K E Gubbins, R Radhakrishnan, and M Sliwinska-Bartkowiak. Effects of Confinement on Freezing and Melting. J. Phys.:
Condens. Matter, 18(6):R15-R68, 2006.

2 X. Jiang, J. Huang, B. G. Sumpter, and R. Qiao. Electro-induced dewetting and concomitant ionic current avalanche in nanopores. J. Phys. Chem. Lett., 4:3120-3126, 


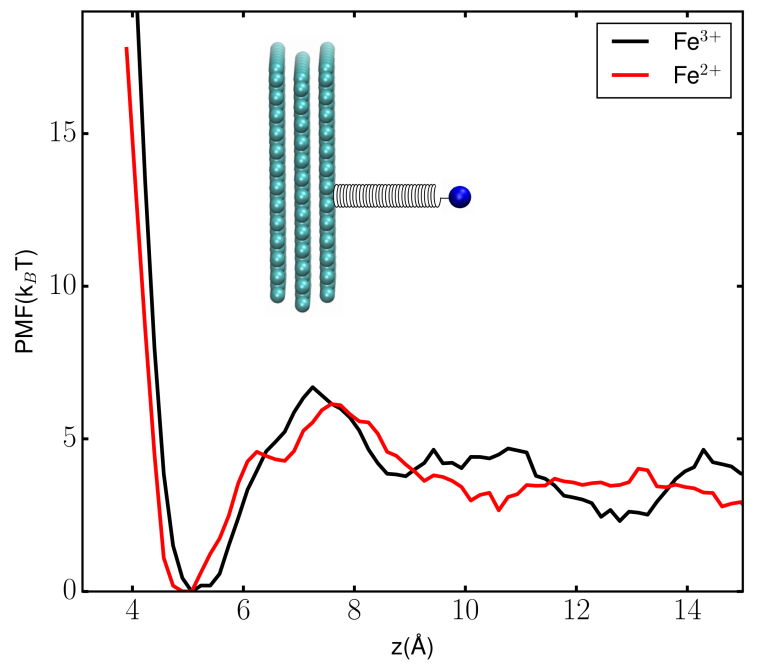

FIG. 9: The potentials of mean force for the $\mathrm{Fe}^{2+}$ and $\mathrm{Fe}^{3+}$ ions approaching the electrode, which are calculated by umbrella sampling method. The $\mathrm{z}$ value indicates the distance between iron and electrode as illustrated in the model of umbrella sampling(inset).

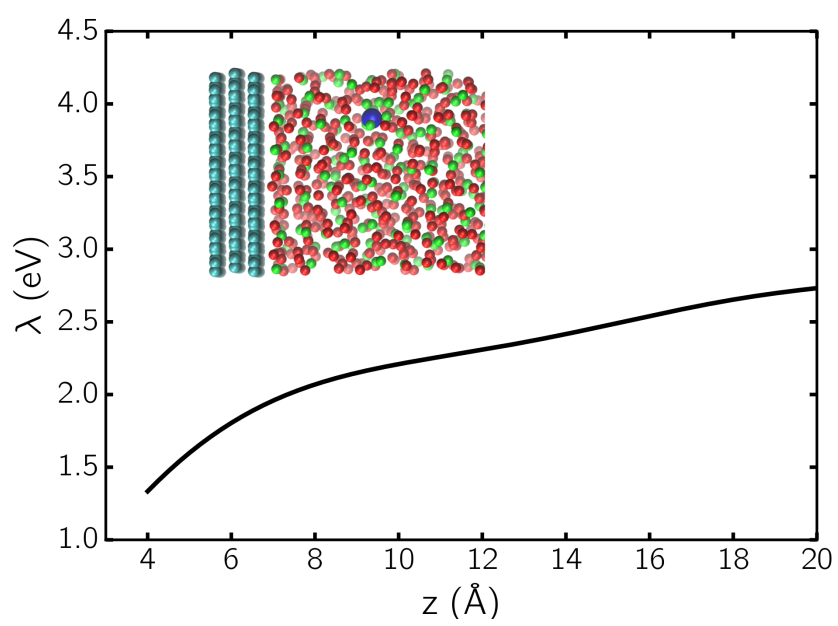

FIG. 10: The calculated reorganization energy of the $\mathrm{Fe}^{2+} / \mathrm{Fe}^{3+}$ redox species using Marcus theory, as a function of distance between iron and electrode.

2013.

3 Clarisse Pean, Barbara Daffos, Benjamin Rotenberg, Pierre Levitz, Matthieu Haefele, Pierre Louis Taberna, Patrice Simon, and Mathieu Salanne. Confinement, Desolvation, and Electrosorption Effects on the Diffusion of Ions in Nanoporous Carbon Electrodes. J. Am. Chem. Soc., 137(39):12627-12632, 2015.

${ }^{4}$ Kumar Varoon Agrawal, Steven Shimizu, Lee W. Drahushuk, Daniel Kilcoyne, and Michael S. Strano. Observation of extreme phase transition temperatures of water confined inside isolated carbon nanotubes. Nat. Nanotech., page doi:10.1038/nnano.2016.254, November 2016.

5 M. Salanne, B. Rotenberg, K. Naoi, K. Kaneko, P.-L.
Taberna, C. P. Grey, B. Dunn, and P. Simon. Efficient storage mechanisms for building better supercapacitors. Nat. Energy, 1:16070, 2016.

6 A. A. Kornyshev. Double-layer in ionic liquids: Paradigm change? J. Phys. Chem. B, 111:5545-5557, 2007.

7 J. Chmiola, G. Yushin, Y. Gogotsi, C. Portet, P. Simon, and P. L. Taberna. Anomalous Increase in Carbon Capacitance at Pore Sizes Less Than 1 Nanometer. Science, 313(5794):1760-1763, 2006.

8 E. Raymundo-Piñero, K. Kierzek, J. Machnikowski, and F. Béguin. Relationship Between the Nanoporous Texture of Activated Carbons and Their Capacitance Properties in Different Electrolytes. Carbon, 44(12):2498-2507, 2006.

9 S. Kondrat, C. R. Perez, V. Presser, Y. Gogotsi, and A. A. Kornyshev. Effect of pore size and its dispersity on the energy storage in nanoporous supercapacitors. Energy Environ. Sci., 5(4):6474-6479, 2012.

10 Cline Merlet, Benjamin Rotenberg, Paul A. Madden, Pierre-Louis Taberna, Patrice Simon, Yury Gogotsi, and Mathieu Salanne. On the molecular origin of supercapacitance in nanoporous carbon electrodes. Nat. Mater., 11(4):306-310, 2012.

11 Y. He, J. Huang, B. G. Sumpter, A. A. Kornyshev, and R. Qiao. Dynamic charge storage in ionic liquids-filled nanopores: Insight from a computational cyclic voltammetry study. J. Phys. Chem. Lett., 6:22-30, 2015.

12 J. Vatamanu and D. Bedrov. Capacitive energy storage: Current and future challenges. J. Phys. Chem. Lett., 6:3594-3609, 2015.

13 Debbie S. Silvester and Richard G. Compton. Electrochemistry in Room Temperature Ionic Liquids: A Review and Some Possible Applications. Z. Phys. Chem, 220(10):1247-1274, oct 2006.

14 Michel Armand, Frank Endres, Douglas R MacFarlane, Hiroyuki Ohno, and Bruno Scrosati. Ionic-liquid Materials for the Electrochemical Challenges of the Future. Nat. Mater., 8(8):621-9, 2009.

15 R. Burt, K. Breitsprecher, B. Daffos, P.-L. Taberna, P. Simon, G. Birkett, X. S. Zhao, C. Holm, and M. Salanne. Capacitance of nanoporous carbon-based supercapacitors is a trade-off between the concentration and the separability of the ions. J. Phys. Chem. Lett., 7:4015-4021, 2016.

16 Eléonore Mourad, Laura Coustan, Pierre Lannelongue, Dodzi Zigah, Ahmad Mehdi, André Vioux, Stefan A. Freunberger, Frédéric Favier, and Olivier Fontaine. Biredox Ionic Liquids with Solid-like Redox Density in the Liquid State for High-energy Supercapacitors. Nat. Mater., 16:446-453, 2017.

17 R. A. Marcus. On the Theory of Oxidation-Reduction Reactions Involving Electron Transfer. I. J. Chem. Phys., 24(5):966-978, 1956.

18 David W. Small, Dmitry V. Matyushov, and Gregory A. Voth. The Theory of Electron Transfer Reactions: What May Be Missing? J. Am. Chem. Soc., 125(24):7470-7478, June 2003.

19 Jochen Blumberger. $\mathrm{Cu}_{\mathrm{aq}}^{+} / \mathrm{Cu}_{\mathrm{aq}}^{2+}$ Redox Reaction Exhibits Strong Nonlinear Solvent Response Due to Change in Coordination Number. J. Am. Chem. Soc., 130(47):1606516068, 2008.

20 Dmitry V. Matyushov and Gregory A. Voth. Modeling the free energy surfaces of electron transfer in condensed phases. J. Chem. Phys., 113(13):5413-5424, October 2000.

21 Rodolphe Vuilleumier, Kafui A. Tay, Guillaume Jeanmairet, Daniel Borgis, and Anne Boutin. Extension of 
Marcus Picture for Electron Transfer Reactions with Large Solvation Changes. J. Am. Chem. Soc., 134(4):2067-2074, feb 2012 .

22 E. Laborda, M. C. Henstridge, C. Batchelor-McAuley, and R. G. Compton. Asymmetric marcus-hush theory for voltammetry. Chem. Soc. Rev., 42:4894-4905, 2013.

23 E. E. L. Tanner, E. O. Barnes, C. B. Tickell, P. Goodrich, C. Hardacre, and R. G. Compton. Application of asymmetric marcus-hush theory to voltammetry in roomtemperature ionic liquids. J. Phys. Chem. C, 119:73607370, 2015.

24 P. G. Dzhavakhidze, A. A. Kornyshev, and L. I. Krishtalik. Activation Energy of Electrode Reactions: the Non-Local Effects. J. Electroanal. Chem., 228:329-346, 1987.

25 D. K. Phelps, A. A. Kornyshev, and M. J. Weaver. Nonlocal Electrostatic Effects on Electron-Transfer Activation Energies: Some Consequences for and Comparisons with Electrochemical and Homogeneous-Phase Kinetics. $J$. Phys. Chem., 94:1454-1463, 1990.

${ }^{26}$ C Merlet, C Péan, B Rotenberg, P. A. Madden, B Daffos, P-L Taberna, P Simon, and M Salanne. Highly Confined Ions Store Charge More Efficiently in Supercapacitors. Nat. Commun., 4(May):2701, 2013.

27 C. Prehal, C. Koczwara, N. Jäckel, A. Schreiber, M. Burian, H. Amenitsch, M. A. Hartmann, V. Presser, and O. Paris. Quantification of ion confinement and desolvation in nanoporous carbon supercapacitors with modelling and in situ x-ray scattering. Nat. Energy, 2:16215, 2017.

28 P. Bai and M. Z. Bazant. Charge transfer kinetics at the solid-solid interface in porous electrodes. Nat. Commun., 5:3585, 2014.

29 B. Akinwolemiwa, C. Peng, and G. Z. Chen. Redox electrolytes in supercapacitors. J. Electrochem. Soc., 162:A5054-A5059, 2015.

30 L.-H. Su, X.-G. Zhang, C.-H. Mi, B. Gao, and Y. Liu. Improvement of the capacitive performances for co-al layered double hydroxide by adding hexacyanoferrate into the electrolyte. Phys. Chem. Chem. Phys., 11:2195-2202, 2009.

31 A. B. Pereiro, J. M. M. Araújo, F. S. Oliveira, J. M. S. S. Esperança, J. N. Canongia Lopes, I. M. Marrucho, and L. P. N. Rebelo. Solubility of Inorganic Salts in Pure Ionic Liquids. J. Chem. Thermodyn., 55:29-36, 2012.

32 Céline Merlet, Mathieu Salanne, and Benjamin Rotenberg. New Coarse-grained Models of Imidazolium Ionic Liquids for Bulk and Interfacial Molecular Simulations. J. Phys. Chem. C, 116(14):7687-7693, 2012.

33 Durba Roy and Mark Maroncelli. An Improved Four-Site Ionic Liquid Model. J. Phys. Chem. B, 114(39):1262912631, October 2010.

34 J. Ilja Siepmann and Michiel Sprik. Influence of surface topology and electrostatic potential on water/electrode systems. J. Chem. Phys., 102(1):511-524, January 1995.

35 J. C. Palmer, A. Llobet, S. H. Yeon, J. E. Fischer, Y. Shi, Y. Gogotsi, and K. E. Gubbins. Modeling the Structural Evolution of Carbide-derived Carbons Using Quenched
Molecular Dynamics. Carbon, 48(4):1116-1123, 2010.

36 Arieh Warshel. Dynamics of Reactions in Polar Solvents. Semiclassical Trajectory Studies of Electron-transfer and Proton-transfer Reactions. J. Phys. Chem., 86(12):22182224, jun 1982.

37 Jenn Kang Hwang and Arieh Warshel. Microscopic Examination of Free-energy Relationships for Electron Transfer in Polar Solvents. J. Am. Chem. Soc., 109(3):715-720, feb 1987.

38 R. A. Marcus. On the Theory of Electron-Transfer Reactions. VI. Unified Treatment for Homogeneous and Electrode Reactions. J. Chem. Phys., 43(2):679-701, jul 1965.

39 Yuri Georgievskii, Chao-Ping Hsu, and R. A. Marcus. Linear Response in Theory of Electron Transfer Reactions as an Alternative to the Molecular Harmonic Oscillator Model. J. Chem. Phys., 110(11):5307-5317, mar 1999.

40 Gregory King and Arieh Warshel. Investigation of the Free Energy Functions for Electron Transfer Reactions. $J$. Chem. Phys., 93(12):8682-8692, dec 1990.

41 J. Blumberger. Recent advances in the theory and molecular simulation of biological electron transfer reactions. Chem. Rev., 115:11191-11238, 2015.

42 Michael A Pounds, Mathieu Salanne, and Paul A Madden. Molecular Aspects of the $\mathrm{Eu}^{3+} / \mathrm{Eu}^{2+}$ Redox Reaction at the Interface Between a Molten Salt and a Metallic Electrode. Mol. Phys., 113(17-18):2451-2462, sep 2015.

43 R. A. Marcus. Reorganization free energy for electron transfers at liquid-liquid and dielectric semiconductorliquid interfaces. J. Phys. Chem., 94(3):1050-1055, February 1990.

44 Richard C. Remsing, Ian G. McKendry, Daniel R. Strongin, Michael L. Klein, and Michael J. Zdilla. Frustrated Solvation Structures Can Enhance Electron Transfer Rates. J. Phys. Chem. Lett., 6(23):4804-4808, dec 2015.

45 T. J. Abraham, D. R. MacFarlane, and J. M. Pringle. Seebeck coefficients in ionic liquids -prospects for thermoelectrochemical cells. Chem. Commun., 47:6260-6262, 2011.

46 C. Merlet, C. Péan, B. Rotenberg, P. A. Madden, P. Simon, and M. Salanne. Simulating supercapacitors: Can we model electrodes as constant charge surfaces? J. Phys. Chem. Lett., 4:264-268, 2013.

47 L. X. Dang. Mechanism and Thermodynamics of Ion Selectivity in Aqueous Solutions of 18-Crown-6 Ether: A Molecular Dynamics Study. J. Am. Chem. Soc., 117:6954-6960, 1995.

48 K. Ando. Solvent Nuclear Quantum Effects in Electron Transfer Reactions. III. Metal Ions in Water. Solute Size and Ligand Effects. J. Chem. Phys., 114:9470-9477, 2001.

49 S. K. Reed, O. J. Lanning, and P. A. Madden. Electrochemical interface between an ionic liquid and a model metallic electrode. J. Chem. Phys., 126:084704, 2007.

${ }^{50}$ Y. Yamato, Y. Katayama, and T. Miura. Effects of the interaction between ionic liquids and redox couples on their reaction entropies. J. Electrochem. Soc., 6:H309-H314, 2013. 